\title{
A Review on COPD Treatment and Management Strategies
}

\author{
Siddhi Vora ${ }^{1}$, Prof. Chintan Shah ${ }^{2}$ \\ Department of Bio-Medical Engineering ${ }^{1,2}$, Government Engineering College ${ }^{1,2}$ \\ Email: siddhivora9@gmail.com ${ }^{1,}$ cpshah@gecg28.ac.in ${ }^{2}$
}

\begin{abstract}
This study has been undertaken to investigate effectiveness of various available treatment and management strategies for the COPD disease. As COPD is the $4^{\text {th }}$ leading cause of death worldwide, the systematic management of disease lead to the reduction of the global burden of disease. COPD is highly characterized by the irreversible airflow obstruction and bronchial obstruction. People with COPD face problems while breathing. COPD is incurable but a preventable disease, so the properly followed treatment and management strategies can slow down the disease growth factor and improve the quality of life. Treatment strategies are available to allow the doctors to manage the disease more efficiently. Pulmonary Rehabilitation, Pharmacological and non- pharmacological treatment are proven to be adequate for treatment of COPD. Inhaled bronchodilator is the key therapy for all COPD patients.
\end{abstract}

Index Terms- Chronic Obstructive Pulmonary Disease (COPD); emphysema; dyspnea; COPD treatment; bronchodilator; tobacco smoking.

\section{INTRODUCTION}

Chronic Obstructive Pulmonary Disease (COPD) is an obstructive type of lung disease. COPD is a common outcome for subjects exhibiting suffering from emphysema or chronic bronchitis. In the condition of Emphysema the alveoli at the end of the bronchioles (smallest air passages of the lungs) are destroyed, typically from smoking. Daily cough and mucus production is the main symptom of chronic bronchitis, which lasts at least for three months a year. Shortness of breath, chest tightness, wheezing, chronic cough are the major signs of COPD disease.

COPD is now become the $4^{\text {th }}$ leading cause of death worldwide and according to WHO report it is estimated to be the $3^{\text {rd }}$ leading cause of death by 2030[1]. Due to environmental changes and increased use of tobacco, rate of disease progression is increased. Cure is not possible for COPD, but the properly maintained treatment and management strategies can slower the progression of COPD.

COPD patient may also experience the condition of exacerbations, during which COPD symptoms become worse. The exacerbations typically occur in response to an environmental insult encountered by the subject. A variety of biological and non-biological environmental insults may cause exacerbations in COPD patients including second hand cigarette smoke, fumes from gasoline, bacteria, and viral infections.

The main objective of this review article is to review the various treatment options available for COPD and their effects on patient's condition.

\section{COPD MANAGEMENT}

\subsection{Accessing Outcomes}

COPD is a preventable but incurable disease. Management strategies can control the COPD growth factor. So it is always advisable to follow the proper management strategies according to patient's condition. Progression of COPD disease lead to decrease in value of FEV1, increases disability and causes death. Best COPD Treatment and management strategies prevent disease progression rate, reduce the chances of exacerbation, and frequency of hospitalizations. Health status and Quality of life can be improved with properly maintained management strategies[2]. Quality of life includes the increased exercise tolerance and reduced mortality.

Treatment of COPD disease includes 5 different aspects, which are smoking cessation, vaccination, Pharmacological Treatment, Non-pharmacological treatment and Oxygen therapy[3].

\subsection{Smoking Cessation}

WHO report states that $12 \%$ of world's smokers are present in India [4]. The Indian Government has implemented a Tobacco control act with a purpose of prevention of tobacco use by children and individuals spread the awareness about the hazardous effect of tobacco and to protect the individuals from tobacco smoke. According to this act children below 18 year cant purchase or contain tobacco products [4], [5]. The smoking in public areas is banned according to the Anti-smoking Act of India. Report of American Lung Society states that almost $25 \%$ smoker who use medications can stay smoke free for 6 months [6]. Smoking cessation can be ${ }^{2}$ tone by using different aspects such as abruptly quitting without assistance, behavioral counseling, medications and cutting down 


\section{E-ISSN: 2321-9637 \\ Available online at www.ijrat.org}

then quitting. Nicotine replacement therapy (NRT), bupropion, varenicline or cytosine are the medications used for smoking cessation [7]. Tobacco smoke includes Nicotine, which is addictive. Nicotine withdrawal is not easy and can only be obtain with multiple trials. US FDA approved 5 drugs (nicotine gum, nicotine inhaler, transdermal nicotine patches, nicotine sprays and nicotine iozenges) that deliver nicotine in the form that does not include the risk of tobacco smoke. Studies show that patients with varenicline have the higher chances of quitting smoking than the patients with bupropion or NRT. According to USFDA the most effective way of smoking cessation is to use the combination of Nicotine patch and bupropion. Varenicline doubled the chances of quitting with compared to placebo, but have the adverse effects of cardiovascular events [8].

\subsection{Vaccination}

Influenza and Pneumococcal are the two mandatory vaccines for COPD patients. According to GOLD criteria influenza is mandatory for all type of patients [3]. PPSV23 (23-valent pneumococcal polysaccharide vaccine) and PCV13 (13-valent conjugated pneumococcal vaccine) are the two Pneumococcal vaccines which are recommended for the patients $\geq 65$ year of age. The PPSV23 is also recommended for the younger COPD patients [3].

\subsection{Non-Pharmacologic Treatment}

\subsubsection{Pulmonary Rehabilitation}

For COPD, Pulmonary Rehabilitation is the most effective non-pharmacologic treatment, but awareness regarding the rehabilitation is still the major issue. Pulmonary Rehabilitation includes patient education, yoga, breathing exercises, psychosocial support, and dietary instructions. Multiple studies note that, this therapy has the considerable effect on quality of life, frequency of hospitalizations, shortness of breath, psychosocial behaviour and exercise tolerance. The experiment of simplified disease management for a 1 year trial has shown the positive impact by reducing the frequent hospitalizations and visits to emergency room during hospitalization. This experiment is tested over 743 patients with severe COPD.[9]

\subsection{Pharmacologic Treatment}

Multiple drugs are available for the treatment of COPD and this all are well described in several guidelines. Guidelines provide great insight into correct usage of this treatment therapy. Bronchodilators are generally preferred for all COPD patients and it is the key treatment for COPD because it extenuate airflow limitation, improve lung function, reduce shortness of breath and increase exercise tolerance [10][11][12]. Short-acting bronchodilators are not preferred for a regular usage. Patient who require Short-acting bronchodilator frequently, are suggested to use long acting bronchodilator. Effect of Short acting b2 agonists (SABA) lasts over 4 to 6 hour, whereas long acting b2 agonists (LABA) lasts over 12 to 24 hour [13][14]. Long acting bronchodilators such as Salmeterol and Formoterol have greater effect on improving FEV1 but does not affect the mortality rate. Experiments shown that LABA has the greatly reduced the rate of exacerbation compared to long acting antimuscarinic antagonists (LAMA) [14]. In terms of health status and lung function ipratropium (LAMA) has shown the greater effect when compared to SABA. Studies also indicate the safer use of anticholinergic drugs in frequent doses. Dryness of mouth is the only limitation associated with this class of agents.

Experiment of Understanding the Potential LongTerm Impacts on Function with Tiotropium (UPLIFT) is done to understand the long term effect of Tiotropium in 5993 patients of severe stage over a time period of 4 years [15]. 5947 Patients are restricted to use tiotropium or placebo only and other 46 patients are instructed to use all the available medications except for inhaled anticholinergics drugs. With compared to placebo, tiotropium does not shown any considerable effect in the value of FEV1 but significantly reduce the rate of exacerbation. Combined Bronchodilator therapy has shown the higher effect compared to single bronchodilator. Combination of bronchodilator is chosen based on different mechanism and duration of effectiveness with lower risk of adverse effect[16]. To improve value of FEV1 and reduce the symptoms combination of SABA and SAMA have given the best results. Other combination of drugs are also available, which are mentioned in GOLD criteria.

Inhaled corticosteroids are used as the next phase of treatment for the COPD patients. Inhaled corticosteroids is generally preferred for patients with moderate to very severe COPD [3]. It is recommended not to use ICS as monotherapy, it should be added with one or more bronchodilator. One recent experiment is done for placebo controlled trials of ICS, and this experiment is concluded with the result of $30 \%$ reduced rate of exacerbations [17]. ICS added to the LABA is proven to be the most effective than either individual component in terms of improved health status and lung function.

If the symptoms are persistent after the treatment with one or more LABA or LAMA or combination of both then Inhaled corticosteroids are preferred. ICS is also recommended for frequent exacerbations. For the patients with severe disease, regular use of ICS lead to 


\section{E-ISSN: 2321-9637 \\ Available online at www.ijrat.org}

the increased risk of Pneumonia [3]. For severe condition, triple therapy ICS, LABA, and LAMA is proven to be more effective compared to combination therapy of ICS and LABA, or LAMA in terms of improved lung function and reduced rate of exacerbation [18].

Observational study called Toward a Revolution in COPD Health (TORCH)[19] is a placebo controlled, multicenter, double-blind, randomized clinical trial. 6112 patients are observed during this trial over a period of 3 year with the combination treatment of salmeterol and fluticasone. With this experiment they have obtained a decreased risk of mortality compared to placebo monotherapy.

Study of Evaluation of COPD Longitudinally to Identify Predictive Surrogate Endpoints (ECLIPSE)[20], concluded that the rate of reduction in value of FEV1 is highly variable over a period of 3 years with increase in magnitude decline in patients with repeated exacerbation, current smoker and patient with emphysema. Although the mean declination rate is inversely related to GOLD criteria [3]. However, UPLIFT and TORCH studies concluded that declination rate is reduced with the use of LABA in patient with moderate stage. So ultimately, bronchodilator is the key treatment to reduce the rate of decline, but the identification of faster declination rate is very difficult and therefore it is advisable to suggest bronchodilator therapy for all stages of COPD patients.

Experimental trial called Investigating New Standards for Prophylaxis in Reducing Exacerbations (INSPIRE) is conducted on 1323 patients over a randomized period of two year. Two different treatment strategies are studied, one is the combination of salmeterol and fluticasone with dosage of 500/ 50 two times per day, and second with tiotropium with dosage of $18 \mathrm{mg}$ per day [21]. After the trial, no significant change in rate of exacerbation is observed with both of this treatment therapy. Many of the patients face problems in receiving Tiotropium and these results in improved health status.

According to GOLD criteria use of ICS is associated with adverse effects such as pneumonia, hoarse voice, oral candidiasis and skin bruising [3]. Oral Glucosteroids are proven to be an important in treatment of exacerbation but they are not much useful in daily chronic treatment of COPD due to higher chances of complications [19], [21]. Decreased functionality, muscle weakness and chances of respiratory failure are the major complications associated with use of oral Glucosteroids. Rate of complications are increased with patients of very severe COPD. One meta analysis [22] indicates that the use of budesonide for 12 months minimized the risk of pneumonia in patient of COPD during that time but increase the infectious exacerbations[23][20][21][24]. So this trial concludes that ICS reduce the non-infectious exacerbation but increase the chances of infectious exacerbation. Earlier to this survey this infectious exacerbations are considered as the event of pneumonia. Although other factors are also responsible for this type of exacerbations and these factors are still the areas of research.

\subsubsection{Theophylline}

Theophylline is widely used for the treatment of chronic diseases due to its cheaper rate [25]. High risk of adverse effects associated with Theophylline, lead to reduce its usage worldwide. Recent studies show that it has the greater anti-inflammatory effects and restore steroid sensitivity. However this area is still under research.

\subsubsection{Treatment of Acute Exacerbation}

Acute exacerbations are the condition that needs an extra and instant attention and are associated with mortality rate during hospitalizations. After the exacerbations, chances of mortality rate with symptoms of respiratoy failure for the next 6 to 12 months are observed between 30 to $40 \%$. Acute exacerbations are difficult to treat in early stage. Inhaled bronchodilators are generally preferred in the condition of acute exacerbation. Other nebulised formulations are also useful for the persons who are critically ill and are not able to generate the adequate amount of flow. Oxygen therapy is used to maintain the level of oxygen in blood. In the condition of acute exacerbation Oral Glucosteroids play a key role. Adverse effects associated with the Glucosteroids are condition of respiratory failure, decreased functionality, and muscle weakness.

\subsection{Oxygen Therapy}

Oxygen Therapy is generally preferred in the case of respiratory failure to increase the chances of survival. Chronic respiratory failure is the condition when lungs are not able to take enough amount of oxygen which leads to reduced blood oxygen level. Condition of Chronic respiratory failure can be either short term or long term. Experiment shows that the oxygen therapy for a $24 \mathrm{hr}$ is more effective than 12 hour in terms of increased survival chances and it is tested over 87 patients for the time period of 3 years [26]. 


\section{Available online at www.ijrat.org}

Table 1 Summary of various COPD Guidelines

\begin{tabular}{|c|c|c|c|}
\hline Agent & ATS/ ERS & GOLD & ACS \\
\hline SABA, SAMA & $\begin{array}{l}\text { Mild } \\
(\mathrm{FEV} 1> \\
80 \%)\end{array}$ & $\begin{array}{l}\text { Stage 1 } \\
(\mathrm{FEV} 1> \\
80 \%)\end{array}$ & $\begin{array}{l}(\mathrm{FEV} 1< \\
80 \%)\end{array}$ \\
\hline LABA, LAMA & $\begin{array}{l}\text { Moderate } \\
(\text { FEV1< } \\
80 \%)\end{array}$ & $\begin{array}{l}\text { Stage } 2 \\
-4 \\
(\mathrm{FEV} 1< \\
80 \%)\end{array}$ & $\begin{array}{l}(\mathrm{FEV} 1< \\
60 \%)\end{array}$ \\
\hline $\begin{array}{l}\text { Combination of } \\
\text { LABA + LAMA }\end{array}$ & $\begin{array}{l}\text { Severe } \\
(\mathrm{FEV} 1< \\
50 \%)\end{array}$ & $\begin{array}{l}\text { Stage } 3 \\
(\mathrm{FEV} 1< \\
50 \%)\end{array}$ & $\begin{array}{l}(\mathrm{FEV} 1< \\
60 \%)\end{array}$ \\
\hline $\begin{array}{l}\text { Combination of } \\
\text { LABA + } \\
\text { LAMA + ICS }\end{array}$ & $\begin{array}{l}\text { Severe, } \\
\text { Very } \\
\text { Severe } \\
(\text { FEV1 < } \\
50 \%)\end{array}$ & $\begin{array}{l}\text { Stage } 3- \\
4(\mathrm{FEV} 1 \\
<50 \%)\end{array}$ & $\begin{array}{l}(\mathrm{FEV} 1< \\
60 \%)\end{array}$ \\
\hline
\end{tabular}

\section{SUMMARY}

The rate of mortality and morbidity due to COPD is going to be increased day by day. Proper and systematic management of COPD disease may lead to slower progression rate. A COPD patient needs to be evaluated on regular basis so that the Pulmonologist can decide their medications and treatment strategies accordingly. This frequent evaluation leads to reduced growth factor of disease. Moreover this directly affects to the rate of mortality. The advantage of these newer therapies ultimately reduces the burden of COPD disease.

\section{Acknowledgments}

I heartily thanks to Prof. Chintan Shah for his support in preparation of this review article.

\section{REFERENCES}

[1] S. Anakal and P. Sandhya, "Clinical Decision Support System for Chronic Obstructive Pulmonary Disease using Machine Learning Techniques," Int. Conf. Electr. Electron. Commun. Comput. Optim. Tech., pp. 0-4, 2017.

[2] V. C. Glaab T, "Outcome measures in chronic obstructive pulmonary disease ( COPD ): strengths and limitations Review," pp. 1-11, 2010.

[3] M. Sigamani, "Global Initiative for Chronic Obstructive Pulmonary Disease, Global strategy for the diagnosis, management and prevention of chronic obstructive pulmonary disease NHLBI/WHO workshop report.," 2013.

[4] J. Kaur and D. C. Jain, "Tobacco Control Policies in India : Implementation and Challenges Tobacco control legislation in India," vol. 55, no. 3, 2011.

[5] R. Article, "Tobacco Control in India - Where are we now ?," vol. 14, no. 1, 2011.

[6] "TREATMENT OF TOBACCO DEPENDENCE AND SMOKING CESSATION METHODS,” pp. $15-40$.

[7] "Smoking Cessation Products Fact Sheet."

[8] Hamilton Health Sciences, "Medications to help you quit smoking," pp. 1-15, 2018.

[9] K. L. Rice et al., "Disease Management Program for Chronic Obstructive Pulmonary Disease A Randomized Controlled Trial."

[10] K. Cahill, S. Lf, and T. Lancaster, "Nicotine receptor partial agonists for smoking cessation ( Review )," no. 6, 2013.

[11] A. Qaseem, T. J. Wilt, S. E. Weinberger, N. A. Hanania, and G. Criner, "Clinical Guideline Diagnosis and Management of Stable Chronic Obstructive Pulmonary Disease: A Clinical Practice Guideline Update from the American College of Physicians, American College of Chest Physicians , American Thoracic Society, and European Respiratory Society," no. April, pp. 179192, 2011.

[12] B. R. Celli and W. Macnee, "Standards for the diagnosis and treatment of patients with COPD : a summary of the ATS / ERS position paper," pp. 932-946, 2004.

[13] F. A. Mcalister, S. F. P. Man, and N. R. Anthonisen, "Contemporary Management of Chronic Obstructive Pulmonary Disease," vol. 290, no. 17, pp. 2301-2312, 2020.

[14] R. A. Wise and D. P. Tashkin, “Optimizing Treatment of Chronic Obstructive Pulmonary Disease: An Assessment of Current Therapies PHARMACOTHERAPY FOR CHRONIC OBSTRUCTIVE PULMONARY DISEASE : EVIDENCE," vol. 120, pp. 4-13, 2007.

[15] S. Menjoge, D. Ph, M. Decramer, D. Ph, and U. Study, "A 4-Year Trial of Tiotropium in Chronic Obstructive Pulmonary Disease," pp. 1543-1554, 2008.

[16] C. F. Sonal Singh, Yoon K. Loke, "Inhaled Anticholinergics and Risk of Major Adverse Cardiovascular Events in Patients With Chronic Obstructive Pulmonary Disease," vol. 300, no. 12, pp. 1439-1450, 2020.

[17] N. R. M. Faarc, "Corticosteroid Therapy and Chronic Obstructive Pulmonary Disease Corticosteroid Risks for Patients With COPD The Evidence Base for Clinical Efficacy of Corticosteroids in COPD," vol. i, pp. 289-296, 2006.

[18] B. R. Alvar Agusti and R. Che, "Global Initiative for Chronic Obstructive Lung Disease: POCKET GUIDE TO COPD DIAGNOSIS, MANAGEMENT, AND PREVENTION," 2017.

[19] P. W. Jones, J. C. Yates, and J. Vestbo, "Salmeterol and Fluticasone Propionate and Survival in Chronic Obstructive Pulmonary 


\section{Available online at www.ijrat.org}

Disease," 2007.

[20] H. O. C. J. Vestbo, W. Anderson, "Evaluation of COPD Longitudinally to Identify Predictive Surrogate End-points (ECLIPSE)," Flagship Scientific Journal of ERS, 2008. .

[21] J. A. Wedzicha et al., "The Prevention of Chronic Obstructive Pulmonary Disease Exacerbations by Salmeterol / Fluticasone Propionate or Tiotropium Bromide," no. February, 2004.

[22] D. D. Sin et al., "Budesonide and the risk of pneumonia : a meta-analysis of individual patient data," Lancet, vol. 374, no. 9691, pp. 712-719, 2009.

[23] Donald P Tashkin, "Budesonide and formoterol in a single pressurized metered-dose inhaler for treatment of COPD," pp. 703-714, 2010.

[24] S. I. Rennard et al., "Efficacy and Tolerability of Budesonide / Formoterol in One Hydrofluoroalkane Pressurized Metered-Dose Inhaler in Patients with Chronic Obstructive Pulmonary Disease Results from a 1-Year Randomized Controlled Clinical Trial," vol. i, no. 5, pp. 549-565, 2009.

[25] D. Singh, J. Brooks, G. Hagan, A. Cahn, and B. J. O. Connor, "Superiority of " " triple "' therapy with salmeterol / fluticasone propionate and tiotropium bromide versus individual components in moderate to severe COPD," pp. 592-598, 2008.

[26] "Long term domiciliary oxygen therapy in chronic hypoxic cor pulmonale complicating chronic bronchitis and emphysema," no. March, 1981. 